OPEN ACCESS

Edited by:

Susmita Chatterjee,

George Institute for Global

Health, India

Reviewed by:

Hon-Yi Shi,

Kaohsiung Medical University, Taiwan

Tsan-Hon Liou,

Taipei Medical University, Taiwan

Manav Vyas,

University of Toronto, Canada

${ }^{*}$ Correspondence:

Li-Fan Liu

lilian@mail.ncku.edu.tw

Specialty section: This article was submitted to Aging and Public Health,

a section of the journal

Frontiers in Public Health

Received: 22 December 2020

Accepted: 18 June 2021

Published: 05 August 2021

Citation:

Liu L-F, Wang W-M and Wang J-D (2021) Functional and Cognitive Impairments Increased Risks of Outcomes of Healthcare Utilization in Patients With Stroke Receiving Home and Community-Based Care in

Front. Public Health 9:644911 doi: 10.3389/fpubh.2021.644911

\section{Functional and Cognitive} Impairments Increased Risks of Outcomes of Healthcare Utilization in Patients With Stroke Receiving Home and Community-Based Care in Taiwan

\author{
Li-Fan Liu ${ }^{1 *}$, Wei-Ming Wang ${ }^{2}$ and Jung-Der Wang ${ }^{3,4}$ \\ ${ }^{1}$ Institute of Gerontology, College of Medicine, National Cheng Kung University, Tainan, Taiwan, ${ }^{2}$ Department of Statistics, \\ College of Management, National Cheng Kung University, Tainan, Taiwan, ${ }^{3}$ Department of Public Health, College of \\ Medicine, National Cheng Kung University, Tainan, Taiwan, ${ }^{4}$ Department of Occupational and Environmental Medicine, \\ National Cheng Kung University Hospital, College of Medicine, National Cheng Kung University, Tainan, Taiwan
}

Aim: Stroke is a leading cause of disability; however, little is known about the outcomes of the utilization of long-term care (LTC) recipients in Taiwan. This study aimed to quantify the burdens of disease of stroke survivors receiving LTC by evaluating the outcomes of their utilization including mortality, readmissions, and re-emergency within 1 year after diagnoses of strokes.

Methods: By interlinkages among the national mortality registry, LTC dataset (LTC-CM), and the National Health Insurance Research Dataset (NHIRD), the outcomes and the factors associated with receiving LTC up to 1 year were explored. Patients were aged 50 years and over with an inpatient claim of the first diagnosis of stroke of intracerebral hemorrhage $(\mathrm{ICH})$ and ischemic stroke during 2011-2016. Outcomes of the healthcare utilization include rehospitalization and re-emergency.

Results: There were 15,662 patients with stroke who utilized the LTC services in the dataset among the stroke population in NHIRD. Stroke survivors receiving LTC showed no difference in clinical characteristics and their expected years of life loss ( $E Y L L=7.4$ years) among those encountered in NHIRD. The LTC recipients showed high possibilities to be rehospitalized and resent to emergency service within 1 year after diagnosis. Apart from the comorbidity and stroke severity, both the physical and mental functional disabilities and caregiving resources predicted the outcomes of the utilization.

Conclusions: For stroke survivors, both severe functional impairments and cognitive impairments were found as important factors for healthcare utilizations. These results regarding reserving functional abilities deserve our consideration in making the decision on the ongoing LTC policy reform in the aged society of Taiwan.

Keywords: long-term care, mortality, rehospitalization, re-emergency, stroke survivors 


\section{INTRODUCTION}

Stroke is a leading cause of disability and morbidity associated with increased economic burden related to acute treatment, poststroke care (PSC), and rehabilitation. Although notable therapeutic advances have contributed to reducing brain damage and disability in patients with stroke (1), many of them are still left with a functional impairment that prevents them from performing basic activities of daily living. Thus, stroke survivors would still face challenges of long-term care (LTC) and continued rehabilitation, which usually result in a tremendous burden.

The Taiwan government launched the LTC 1.0 policy in 2007, which aimed at assisting frail elderly people with LTC needs. The initial objective was to develop a system of home and community-based services (HCBS), including home services, adult day care, home nursing care, home and community-based rehabilitation, home meal delivery, palliative care for caregivers, and transportation services. To facilitate service delivery, the government began to transform the LTC 1.0 into a new reform of the LTC 2.0 policy system in 2016 (2). Under LTC 2.0 policy, more transitional care linking with LTC has been provided, such as planning for home discharge in the hospitals and home healthcare. However, little has been evaluated about the outcomes of the utilization of LTC recipients under the LTC 1.0 policy. By interlinkages among the national mortality registry, LTC dataset (LTC-CM), and the National Health Insurance Research Dataset (NHIRD), this study aimed to quantify the burdens of disease of patients with different subtypes of strokes under LTC by evaluating their mortality and outcomes of the utilization including readmissions and re-emergency within 1 year after the diagnoses of strokes under the National Health Insurance (NHI). The results of these real-world data could provide additional evidence for improving service delivery and reform of the LTC policy 2.0 in Taiwan.

\section{Literature Review}

The risk of stroke increases with age, is more common among women, and is a major contributor to long-term disability, especially among the elderly (3). Previous studies in the US indicated that nearly $90 \%$ of strokes are ischemic, which are caused by a blockage in the cerebral artery, which restricts blood flow (4). Based on the International Classification of Diseases, Ninth Revision, Clinical Modification (ICD-9-CM), the incidence of stroke admissions was high among the acute ischemic stroke (AIS) and intracerebral hemorrhage (ICH).

Caring for stroke survivors imposes a substantial economic burden on society (3). The activities of daily living (basic and instrumental) are reliable indicators of the functional status of patients affected by stroke for the initial evaluation (5). An Australian study showed that among 3-month stroke survivors, $74 \%$ required assistance with activities of daily living and received informal care from family or friends (6). In the U.S., $75 \%$ of survivors returned home 1 year after index stroke admission (7). In Taiwan, an earlier study showed that about $15.2 \%$ were institutionalized after 6 months of stroke (8).

Stroke survivors also suffered from the heavy burden of disease. In the US, stroke is one of the top ten contributors to Medicare costs (4). Taiwan has had a well-established NHI system with over a 99\% coverage rate for acute care since 1995 . It is estimated that in Taiwan, nearly $75 \%$ of those who need LTC are aged 65 years and over (9). Although the recipients of such LTC program must be reassessed every 6 months under the regulations of 1.0 LTC policy, to the best of our knowledge, the outcomes of the utilization have not been systematically evaluated for continued quality assurance, improvement of the care plan, and possible development into an insurance system.

Therefore, from the perspective of LTC, this study explored the profile of stroke survivors who utilized formal HCBS in Taiwan and examined their 1-year outcomes and the predicting factors after index stroke admission and receiving LTC. In this study, we summarized the profile (sociodemographics and functional disabilities) of the LTC recipients with stroke during 2011-2016 among those encountered in NHI of Taiwan, including their life expectancy (LE) and expected years of life loss (EYLL). Then, LTC recipients were enrolled for the analyses of the outcomes related to healthcare utilizations, such as rehospitalization and re-emergency up, to 1 year after index stroke admission and the associated predicting factors. With evidence coming from these real-world data, we hope to add information regarding health outcomes of stroke care in HCBS and hope to be useful to sustain stroke care under the LTC system in Taiwan.

\section{MATERIALS AND METHODS}

The study protocol (no: A-ER-106-183) was approved by the Institution Review Board before commencement, and no conflicts of interest exist between the authors and the goals of this study.

\section{Long-Term Care Data Set}

The data were obtained from the national LTC-CM in Taiwan. This dataset has been maintained by the LTC center in each county, in which the health and functional indicators of care recipients are recorded in an initial needs assessment and are reassessed by care managers during the follow-up process (10, 11). This study is designed to interlink records of the LTC-CM during 2011-2016 with NHIRD and to explore the outcome of healthcare utilization and the factors associated with receiving HCBS up to 1 year.

\section{Case Selection}

The inclusion criteria were patients aged 50 years and over with an inpatient claim of the first diagnosis of stroke defined as follows: confirming principal diagnosis codes for primary or secondary ICD-9 codes of ICH (431.x) and ischemic stroke (433.x and 434.x) from January 2011 to December 2016, in any position by ICD-9-CM and its matched codes in ICD-10 since 2016. The flowchart of the study population is shown in Figure $\mathbf{1}$.

To identify the LTC recipients with diagnoses of strokes mentioned above, cases were further identified by linking the LTC dataset with NHIRD. In total, there were 249,233 cases identified, and among them 15,662 persons received HCBS in the LTC system of Taiwan after discharge from hospitals. 


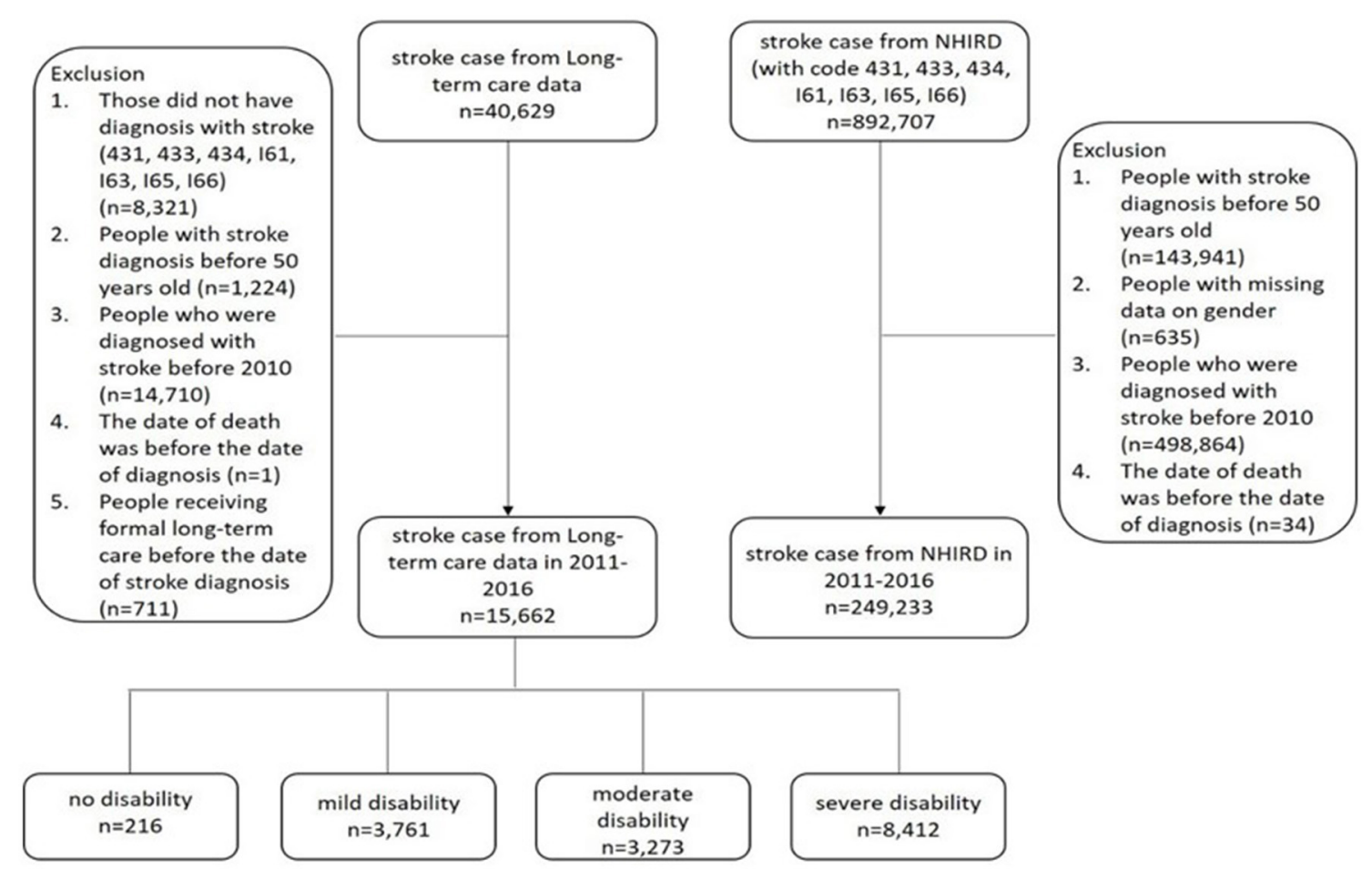

FIGURE 1 | Flow chart of the study samples.

\section{Statistical Analysis}

For the analyses of the predictors of healthcare utilizations (rehospitalization and re-emergency) up to 1 year, the negative binomial regression models were constructed and the dependent variables are a count of events by controlling the time periods staying in the HCBS system. The analyses were performed using Statistical Analysis System (SAS) version 9.4.

After verifying the survival status of all cases through crossreferencing with the national mortality registry at the end of the follow-up period (December 31,2016), the Kaplan-Meier method was used to estimate the survival function based on the 20112016 follow-up data. Extrapolation of survival to lifetime for the stroke cohort after the termination of the follow-up period was conducted. Because the LE is the summation of the area under the lifetime survival curve, we would be able to obtain the EYLL by comparing the LEs between the stroke cohort and that of the correspondingly matched referents $(12,13)$. The approach was mathematically proved valid for predicting the LE under the assumption of constant excess hazard earlier (14-16). Moreover, the software has been updated in the iSQoL 2 with a new algorithm of "rolling over" month by month and only assumes constant hazard within the extrapolated 1 month to accommodate diseases with versatile courses, such as stroke (12).

\section{Measures of Stroke Severity and Comorbidities}

In this study, stroke severity index (SSI) and modified Charlson comorbidity index (modified CCI) were used to identify stroke severity and comorbidities. A claims-based SSI is found to be a valid proxy for the National Institutes of Health Stroke Scale (NIHSS) and an effective adjustment for stroke severity in studies of AIS or ICH outcome with administrative claims data (17-19).

The severity of comorbidities was summarized using a modified version of the CCI that excluded cerebrovascular disease and hemiplegia (20). The modified CCI was dichotomized into low comorbidity $(\leq 1)$ or high comorbidity $(>1)$ for analyses.

\section{RESULTS}

There were 15,662 patients with stroke who utilized the HCBS after the selection process of patients. The detailed profiles of the LTC recipients with stroke from 2011 to 2016 are summarized in Table 1. It showed that the average age of women was higher than men. The ratio of ischemic stroke vs. hemorrhagic stroke was slightly higher than 4:1, and the mean age of those with a higher SSI was younger. More than half (53.1\%) of them entered into the LTC system $<6$ months after stroke admission. Approximately $19.1 \%$ of the LTC recipients were from mid-low/low-income households, and more than $90 \%$ of them were accompanied by caregivers at own home at the first entry to LTC.

In terms of self-care functions, more than half of them (53.7\%) were suffering from severe difficulties in activities of daily living (ADLs <30). About $63.9 \%$ of the LTC recipients showed a score of instrumental activities of daily living (IADL) lower than 8 . Based on the thresholds of the Center for Epidemiologic Studies Depression (CESD), $31.8 \%$ of them seemed depressive and about 
TABLE 1 | The profile (sociodemographics and functional disabilities) of the long-term care (LTC) recipients with stroke from 2011 to 2016.

\begin{tabular}{|c|c|c|c|}
\hline & $N(\%)$ & Age & $p$-value \\
\hline Age (years) & $15,662(100 \%)$ & $73.7 \pm 9.4$ & \\
\hline \multicolumn{4}{|l|}{ Gender } \\
\hline Male & $8,702(55.6 \%)$ & $72.0 \pm 9.7$ & $<0.001$ \\
\hline Female & $6,960(44.4 \%)$ & $75.1 \pm 8.6$ & \\
\hline \multicolumn{4}{|l|}{ Stroke subtype } \\
\hline Hemorrhagic Stroke & 2,921 (18.6\%) & $69.3 \pm 10.3$ & $<0.001$ \\
\hline Ischemic Stroke & $12,741(81.4 \%)$ & $74.3 \pm 8.9$ & \\
\hline \multicolumn{4}{|l|}{ Stroke severity index } \\
\hline Mild $\leq 5$ & $5,611(35.8 \%)$ & $73.6 \pm 8.8$ & $<0.001$ \\
\hline Moderate 5-13 & $4,660(29.8 \%)$ & $74.0 \pm 9.1$ & \\
\hline Severe $>13$ & $5,391(34.4 \%)$ & $72.5 \pm 10.1$ & \\
\hline \multicolumn{4}{|l|}{ Time to long-term care } \\
\hline $0-6$ & $8,311(53.1 \%)$ & $75.5 \pm 8.4$ & $<0.001$ \\
\hline $6-12$ & $2,244(14.3 \%)$ & $71.2 \pm 10.1$ & \\
\hline $12-24$ & $2,234(14.3 \%)$ & $71.0 \pm 10.1$ & \\
\hline $24+$ & $2,873(18.3 \%)$ & $70.8 \pm 9.5$ & \\
\hline \multicolumn{4}{|l|}{ Welfare status } \\
\hline Non-low-income households & $12,673(80.9 \%)$ & $74.7 \pm 8.7$ & $<0.001$ \\
\hline Mid-low/low incomes households & 2,989 (19.1\%) & $67.8 \pm 10.2$ & \\
\hline \multicolumn{4}{|l|}{ Caregivers } \\
\hline Yes & 14,403 (92.0\%) & $73.4 \pm 9.4$ & 0.005 \\
\hline No & $1,259(8.0 \%)$ & $72.6 \pm 9.6$ & \\
\hline \multicolumn{4}{|l|}{$\mathrm{ADL}^{\dagger}$ difficulties } \\
\hline Severe (score 0-30) & $8,412(53.7 \%)$ & $75.2 \pm 8.9$ & $<0.001$ \\
\hline Moderate (score 31-60) & $3,273(20.9 \%)$ & $72.8 \pm 9.4$ & \\
\hline Mild (score 61-100) & 3,977 (25.4\%) & $70.7 \pm 9.5$ & \\
\hline \multicolumn{4}{|l|}{ IADL $^{\ddagger}$ difficulties } \\
\hline Severe (score <8) & $10,010(63.9 \%)$ & $74.3 \pm 9.1$ & $<0.001$ \\
\hline Mild (score $\geq 8$ ) & 5,652 (36.1\%) & $71.7 \pm 9.6$ & \\
\hline \multicolumn{4}{|l|}{ CESD ${ }^{\S}$} \\
\hline No depressive symptoms & $10,684(68.2 \%)$ & $72.5 \pm 9.4$ & $<0.001$ \\
\hline Depressive & $4,978(31.8 \%)$ & $75.2 \pm 9.1$ & \\
\hline \multicolumn{4}{|l|}{ MDAII $^{\mathbb{I}}$ cognition } \\
\hline Severe impairment & $5,446(34.8 \%)$ & $75.8 \pm 8.9$ & $<0.001$ \\
\hline Moderate impairment & 2,399 (15.3\%) & $74.7 \pm 8.7$ & \\
\hline Mild impairment & 1,972 (12.6\%) & $73.4 \pm 9.1$ & \\
\hline No impairment & 5,848 (37.3\%) & $70.5 \pm 9.4$ & \\
\hline
\end{tabular}

${ }^{\dagger} A D L$, activity of daily living.

${ }^{\ddagger} I A D L$, Instrumental activity of daily living.

${ }^{\S}$ CESD, Center for Epidemiologic Studies Depression, the thresholds of depressive tendency: male $\geq 12$, female $\geq 10$.

IMDAI, multi-dimensional assessment instrument for cognition.

$34.8 \%$ of the LTC recipients showed severe cognitive impairment (Table 1).

Table 2 shows the clinical characteristics of stroke patients receiving LTC among those encountered in NHI of Taiwan. The mean age of the LTC recipients with stroke was significantly older than their counterparts in NHIRD [73.7 \pm 9.4 vs. $70.7 \pm$ 10.6 , standardized difference $(\mathrm{SD})=0.3]$. Alternatively, all the calculated SDs of comorbidities and severity between patients
TABLE 2 | The prevalence and means of clinical characteristics at baseline of patients with stroke receiving LTC among those hospitalized through National Health Insurance (NHI) of Taiwan during 2011-2016.

\begin{tabular}{|c|c|c|c|}
\hline Clinical characteristic & $\begin{array}{l}\text { LTC recipients } \\
\qquad(n=15,662)\end{array}$ & $\begin{array}{c}\text { NHI } \\
(n=249,233)\end{array}$ & SMD \\
\hline Age (years), Mean (SD) & $73.7 \pm 9.4$ & $70.7 \pm 10.6$ & $0.30^{\star}$ \\
\hline Sex (Female), $N(\%)$ & $6,960(44.4)$ & 103,703 (41.6) & 0.06 \\
\hline \multicolumn{4}{|l|}{ Stroke subtype } \\
\hline Hemorrhagic, $N(\%)$ & 2,921 (18.6) & 43,962 (17.6) & 0.03 \\
\hline Ischemic, $N(\%)$ & $12,741(81.4)$ & $205,271(82.4)$ & \\
\hline \multicolumn{4}{|l|}{ Modified $\mathrm{CCl}^{\dagger}$} \\
\hline 0 or $1, N(\%)$ & $9,885(63.1)$ & $157,050(63.0)$ & $<0.01$ \\
\hline$>1, N(\%)$ & 5,777 (36.9) & $92,183(37.0)$ & \\
\hline Hypertension, N (\%) & $12,212(78.0)$ & $185,410(74.4)$ & 0.08 \\
\hline Diabetes mellitus, $N(\%)$ & $6,287(40.1)$ & $94,626(38.0)$ & 0.04 \\
\hline Hyperlipidemia, N (\%) & $5,300(33.8)$ & 89,668 (36.0) & 0.04 \\
\hline Prior stroke, $N(\%)$ & $3,518(22.5)$ & $64,392(25.8)$ & 0.08 \\
\hline Atrial fibrillation, $N(\%)$ & 2,324 (14.8) & $28,563(11.5)$ & 0.10 \\
\hline Coronary heart disease, $N(\%)$ & 1,689 (10.8) & 26,952 (10.8) & $<0.01$ \\
\hline Chronic kidney disease, $N(\%)$ & $1,312(8.4)$ & 24,393 (9.8) & 0.05 \\
\hline $\mathrm{SSI}^{\ddagger}$ score & $10.2 \pm 6.2$ & $9.1 \pm 6.2$ & 0.18 \\
\hline Hemorrhagic Stroke & $15.6 \pm 5.2$ & $14.8 \pm 5.9$ & 0.14 \\
\hline Ischemic stroke & $9.0 \pm 5.7$ & $7.9 \pm 5.6$ & 0.19 \\
\hline Life expectancy (in year) & $6.12 \pm 0.12$ & $8.08 \pm 0.10$ & $17.74^{*}$ \\
\hline $\begin{array}{l}\text { Expected years of life loss (in } \\
\text { year) }\end{array}$ & $7.38 \pm 0.14$ & $7.36 \pm 0.10$ & 0.17 \\
\hline
\end{tabular}

${ }^{\dagger} \mathrm{CCl}$, Charlson comorbidity index.

$¥ \mathrm{SSI}$, stroke severity index.

"SMD, standardized mean difference is usually considered statistically significant if $>0.2$.

with stroke receiving LTC and those encountered in the NHI were below 0.2. For example, the two groups looked similar. Although the LE of those receiving LTC was 1.96 years lower than that of the NHI patients with stroke, there was no difference in their EYLL, or both cohorts lost about 7.4 years of LE. It indicates that the difference of their LE would be from a different age of onset.

Table 3 summarizes the 1-year mortality, rehospitalization, and re-emergency among the LTC recipients. We found that the mortality rate of the LTC cohort seemed to be generally low $(8.4 \%)$ during the first year. However, the proportion of rehospitalization seemed high. It showed the proportion of about $30.7 \%$ within 1 month and $63.2 \%$ within 1 year among LTC recipients. The 1-year average times of rehospitalization were also significantly higher than the nationwide cohort $(1.69 \pm$ 2.21 vs. $1.02 \pm 1.76$ ), especially among those with hemorrhagic stroke. The proportion of re-emergency within 1 month appeared similar between the two cohorts ( 14.6 vs. $12.8 \%$ ), the difference increased gradually along time, and the average frequency of re-emergency at the end of 1 year for LTC recipients was significantly higher than that of the nationwide cohort (1.65 \pm 2.46 vs. $1.01 \pm 2.09$ ).

Table 4 presents the inferential analyses for predictors of rehospitalization and re-emergency up to 1 year among the LTC 
TABLE 3 | The mortality and follow-up indices (rehospitalization and re-emergency) of patients with stroke receiving LTC among the National Health Insurance Research Dataset (NHIRD) in Taiwan during 2011-2016.

\begin{tabular}{|c|c|c|c|c|}
\hline Characteristic & LTC recipients $(n=15,662) N(\%)$ & NHIRD $(n=249,233) N(\%)$ & $p$-value & SMD \\
\hline Age (years) & $73.7 \pm 9.4$ & $70.7 \pm 10.6$ & & $0.30^{*}$ \\
\hline \multicolumn{5}{|l|}{ Mortality } \\
\hline$<1$ month & $44(0.3)$ & $21,611(8.7)$ & $<0.001$ & $0.41^{*}$ \\
\hline $1-3$ months & $238(1.5)$ & $9,976(4.0)$ & $<0.001$ & 0.15 \\
\hline 3-6 months & 365 (2.3) & $7,290(2.9)$ & $<0.001$ & 0.04 \\
\hline 6-12 months & $673(4.3)$ & $9,833(4.0)$ & $<0.001$ & 0.02 \\
\hline Total (0-12 months) & $1,320(8.4)$ & $48,710(19.5)$ & $<0.001$ & 0.02 \\
\hline \multicolumn{5}{|l|}{ Re-hosp. } \\
\hline$<1$ month & 4,795 (30.7) & 36,359 (15.9) & $<0.001$ & $0.35^{\star}$ \\
\hline $1-3$ months & 4,812 (30.7) & 48,268 (19.4) & $<0.001$ & $0.26^{\star}$ \\
\hline 3-6 months & 4,413 (28.2) & 42,108 (16.9) & $<0.001$ & $0.27^{\star}$ \\
\hline $6-12$ months & 4,897 (31.3) & $48,547(19.5)$ & $<0.001$ & $0.27^{\star}$ \\
\hline Total (0-12 months) & 9,902 (63.2) & $110,182(44.2)$ & $<0.001$ & $0.39^{*}$ \\
\hline 1-year average & $1.69 \pm 2.21$ & $1.02 \pm 1.76$ & & $0.34^{*}$ \\
\hline Hemorrhagic Stroke & $2.21 \pm 2.68$ & $1.19 \pm 2.08$ & $<0.001$ & $0.43^{\star}$ \\
\hline Ischemic stroke & $1.58 \pm 2.07$ & $0.98 \pm 1.68$ & $<0.001$ & $0.32^{*}$ \\
\hline \multicolumn{5}{|l|}{ Re-ER } \\
\hline$<1$ month & 2,276 (14.6) & $29,040(12.8)$ & $<0.001$ & 0.05 \\
\hline 1-3 months & 3,802 (24.3) & 39,737 (15.9) & $<0.001$ & $0.21^{*}$ \\
\hline 3-6 months & 4,034 (25.8) & 40,649 (16.3) & $<0.001$ & $0.23^{*}$ \\
\hline 6-12 months & $5,497(35.1)$ & $54,736(22.0)$ & $<0.001$ & $0.29^{*}$ \\
\hline Total (0-12 months) & $9,067(57.9)$ & $109,145(43.8)$ & $<0.001$ & $0.28^{\star}$ \\
\hline 1-year average & $1.65 \pm 2.46$ & $1.01 \pm 2.09$ & $<0.001$ & $0.28^{*}$ \\
\hline Hemorrhagic Stroke & $1.54 \pm 2.32$ & $0.80 \pm 1.73$ & $<0.001$ & $0.36^{*}$ \\
\hline Ischemic stroke & $1.67 \pm 2.48$ & $1.06 \pm 2.16$ & $<0.001$ & $0.26^{*}$ \\
\hline
\end{tabular}

"Standardized mean difference (SMD) is usually considered statistically significant if >0.2.

recipients with stroke $(n=12,789)$. Based on the construction of negative binomial regression models, we found that men, high severity, more comorbidity, and those without caregivers were more likely to be readmitted, even after adjustment for multiple functional disabilities. Functional disabilities including ADLs, IADLs, and cognitive impairments were significant predictors for rehospitalization in the follow-up of 1 year. For the re-emergency rates (Table 4), the results again showed that functional disabilities including severe dependence on ADLs, CESD, and severe cognitive impairments were significant predictors for re-emergency in the follow-up of 1 year. That is, subjects with severe functional disabilities of self-care, daily activities, cognition, and depression would be more likely to be rehospitalized or sent to the emergency by the end of the follow-up of 1 year.

\section{DISCUSSIONS}

Stroke is a major contributor to long-term functional disability, especially among the elderly $(3,21)$. For stroke survivors, the need for LTC seems inevitable. The study explored the profile of stroke survivors who utilized formal HCBS of Taiwan and followed their utilization outcomes up to 1 year. The influencing factors of utilization outcomes after index stroke admission were also examined.

The LTC policy in Taiwan has been launched by the central government since 2007 aiming at developing the HCBS. For stroke survivors, apart from institutional care, home nursing and home services remained the primary LTC services during the time period of the study. However, among stroke survivors with LTC needs, how many received the help from the HCBS and how were their healthcare utilization outcomes and predictors have not yet been explored and somehow ignored due to the integrating gap between acute care and LTC. Although the post-acute care plan has been launched in a few local hospitals during the follow-up time since 2014, the outcomes of the utilization of stroke survivors receiving the HCBS afterward and the predictors would be important to effort improvement on clinical interventions and the ongoing LTC policymaking. To the best of our knowledge, the outcomes of the utilization of stroke survivors in receiving the HCBS up to 1 year and the predictors were first analyzed in Taiwan.

Previous studies showed that $<9 \%$ of patients with AIS received long-term home care after hospitalization in the US (21). Our results found that for the patients with ICH and AIS, there was about $6.3 \%$ of the stroke survivors who used the HCBS after 
TABLE 4 | Estimates of incidence rate ratio (IRR) with 95\% Cl for risk factors of 1-year outcomes (rehospitalization, re-emergency) among the LTC recipients with stroke based on negative binomial regressions.

\begin{tabular}{|c|c|c|}
\hline Risk factors & $\begin{array}{c}\text { Times of } \\
\text { Re-hospitalization in } \\
1 \text { year } \\
\text { IRR }(95 \% \mathrm{Cl})\end{array}$ & $\begin{array}{c}\text { Times of } \\
\text { Re-emergency in } \\
1 \text { year } \\
\text { IRR }(95 \% \mathrm{Cl})\end{array}$ \\
\hline Age & $0.98(0.98,0.98)^{\star \star \star}$ & $1.01(1.00,1.01)^{\star \star \star}$ \\
\hline Female (vs. Male) & $0.81(0.78,0.81)^{\star \star \star}$ & $0.85(0.81,0.89)^{\star \star \star}$ \\
\hline Mid/low vs. non-low incomes & $0.92(0.87,0.97)^{\star \star}$ & $1.00(0.94,1.06)$ \\
\hline $\begin{array}{l}\text { No Caregivers (vs. with } \\
\text { caregivers) }\end{array}$ & $1.14(1.06,1.24)^{\star \star \star}$ & $1.16(1.06,1.26)^{\star \star \star}$ \\
\hline $\begin{array}{l}\text { Hemorrhagic Stroke (vs. } \\
\text { Ischemic Stroke) }\end{array}$ & $1.01(0.96,1.07)$ & $0.95(0.89,1.01)$ \\
\hline Charlson comorbidity index & $1.05(1.04,1.07)^{\star \star \star}$ & $1.09(1.08,1.11)^{\star \star \star}$ \\
\hline Stroke severity index & $1.03(1.03,1.03)^{\star \star \star}$ & $1.00(0.99,1.00)$ \\
\hline \multicolumn{3}{|l|}{ Functional disability } \\
\hline $\mathrm{ADL}^{\dagger}$ severe $\left(\mathrm{vs} . \mathrm{ADL}^{\dagger}\right.$ mild $)$ & $1.54(1.45,1.65)^{\star \star \star}$ & $1.47(1.36,1.57)^{\star \star \star}$ \\
\hline $\mathrm{ADL}^{\dagger}$ moderate (vs. $\mathrm{ADL}^{\dagger}$ mild) & $1.24(1.16,1.32)^{\star \star \star}$ & $1.07(0.99,1.15)$ \\
\hline IADL severe (vs. IADL ${ }^{\ddagger}$ mild) & $1.05(1.00,1.10)^{\star}$ & $1.05(0.99,1.10)$ \\
\hline $\begin{array}{l}\text { CESD }{ }^{\S} \text { depression (vs. no } \\
\text { depression) }\end{array}$ & $1.02(0.97,1.08)$ & $1.08(1.02,1.14)^{\star \star}$ \\
\hline $\begin{array}{l}\text { Cognition impairment mild (vs. } \\
\text { no imp.) }\end{array}$ & $0.98(0.92,1.05)$ & $1.05(0.98,1.13)$ \\
\hline $\begin{array}{l}\text { Cognition impairment moderate } \\
\text { (vs. no. imp.) }\end{array}$ & $1.02(0.96,1.09)$ & $1.07(0.99,1.15)$ \\
\hline $\begin{array}{l}\text { Cognition impairment severe (vs. } \\
\text { no imp.) }\end{array}$ & $1.12(1.05,1.20)^{\star \star \star}$ & $1.19(1.11,1.28)^{\star \star \star}$ \\
\hline
\end{tabular}

${ }^{\dagger} A D L$, activity of daily living.

${ } I A D L$, Instrumental activity of daily living.

${ }^{\S}$ CESD, Center for Epidemiologic Studies Depression, the thresholds of depressive tendency: male $\geq 12$, female $\geq 10$.

${ }^{*} p<0.05,{ }^{* *} p<0.01$, and ${ }^{* \star *} p<0.001$.

index stroke admission during the study period. The utilization rate could be influenced by many contextual factors in various countries. In Taiwan, the development of LTC infrastructure and lack of workforce may cause the low utilization rates during the study period. Some other factors from the demand side may be also influential, such as in Taiwan, preference of families of hiring foreign care workers at own homes to support informal caregiving instead of using formal HCBS subsidized by the central government $(10,22)$.

In general, we found no difference between stroke survivors at baseline among who utilized LTC or not after index stroke admission in NHIRD. Regarding LE and EYLL, the LTC recipients were found to be those with shorter LE that may be partly explained by their higher mean age. However, there were no differences in EYLL between the two groups in long term.

For the mortality rates, the result showed that the LTC recipients were those stroke survivors with lower percentages of mortality, especially within 1 month of occurring stroke. The mortality rates among the LTC recipients had increased gradually after index stroke admission, however, showing no statistical difference with their counterparts nationwide after 1 month.
Therefore, it seems not surprised that those who utilized LTC or not showed no influence in terms of EYLL and mortality.

Regarding the utilizations, all-cause readmission rates and re-emergency rates within 1 year of utilizations of HCBS had been focused on and analyzed in this study. The higher rehospitalization and re-emergency rates and the 1-year average of utilizations among the LTC recipients were, however, significantly higher than their counterparts nationwide. A previous study on poststroke in Singapore showed that the highest utilization occurred in the first quarter poststroke across all service types and decreased with time since stroke (23). This study found that the percentage of rehospitalization and re-emergency among patients with stroke has increased within 1-year follow-up. If the results mean worse outcomes or because of higher accessibilities of healthcare utilizations after receiving HCBS was not examined in this study and needs to be further addressed. However, these results deserve our notice and consideration in making the decision on national health and LTC policy, especially on prevention.

Few studies have systematically evaluated predictors of readmission after stroke, a measure of inpatient quality of care (24). The previous study indicated that the use of care after hospitalization for AIS was greatest for individuals with more severe strokes, lower functioning at hospital discharge, older age, unmarried, and black in the US (21). This study has shown some key predictors for outcomes of the utilization up to 1 year after index stroke admission. For the rehospitalization, apart from the sociodemographic factors of the LTC recipients, the modified CCI and SSI predicted the utilization. For the re-emergency, the result showed that hemorrhagic stroke has less incidence rate ratio (IRR) than ischemic stroke for re-emergency in the study. The findings also showed the consistency that the modified CCI was a significant predictor for subsequent rehospitalization and re-emergency within 1-year follow-up.

Moreover, the levels on functional disabilities including both physical (ADLs and IADLs) and mental (CESD and cognition status) were found to be key predictors in the outcomes of the utilization (rehospitalization and re-emergency). The study indicated that functional impairment is associated with increased risk of 30-day, all-cause hospital readmission in Medicare seniors, especially those admitted for heart failure, myocardial infarction, or pneumonia (25). Our study found that the influence exists after the follow-up up to 1 year after index stroke admission. Both the physical and the mental functional impairments were influential for the rehospitalization and re-emergency, especially for those in severe conditions. For senior stroke survivors in Taiwan, functional impairment and severe cognitive impairment were found as important factors in preventing readmissions, and severe functional impairment, depression, and severe cognitive impairment were found as important factors in preventing re-emergency.

Our results reflect the notion proposed by the WHO that the composite of all the physical and mental capacities of an individual at any point in time, or intrinsic capacity, is important. Both the health and social care services should be targeted toward preventing and managing declines in intrinsic capacity and improving functional ability in the elderly $(26,27)$. For 
example, LTC would be a means to ensure and facilitate the elderly with a significant loss of capacity for healthy aging through reengineering and reconstructing their functional ability and intrinsic capacity.

As part of the learning process, this study examined the outcomes of services of patients with stroke receiving the HCBS and hoped to be useful to facilitate the policy to provide closer continuity of LTC services with quality.

\section{Limitations}

Some limitations in the study need to be addressed. First, to narrow down stroke survivors, only patients with ICH and AIS with admission index from NHIRD with or without LTC use were identified in the study by linking the LTC dataset. Patients with stroke who were not hospitalized or treated at outpatient clinics were not included in the study. Because patients with a definite diagnosis of stroke can be waived from copayment for the first month in the NHI system of Taiwan, our collection would include almost all patients with stroke except those with minimal transient ischemic attack and would be relatively representative. Second, only the LTC dataset was available with information of functional disabilities of patients and no such data collected in NHIRD. Thus, our regression models could only present those ever-received services from LTC. Finally, we chose to follow up on 1-year outcomes of the healthcare utilization in the study instead of a lifelong perspective. Future studies of a more comprehensive evaluation of outcome, including long-term survival and dynamic changes of functional disabilities, would be warranted to assess the cost-effectiveness of such services.

\section{CONCLUSIONS}

In Taiwan, stroke survivors receiving HCBS showed no difference in clinical characteristics from NHIRD nationwide. For them,

\section{REFERENCES}

1. Montagu A, Reckless IP, Buchan AM. Stroke: management and prevention. Medicine. (2012) 40:490-9. doi: 10.1016/j.mpmed.2012.06.007

2. Ministry of Health and Welfare. Ten-Year Long-Term Care Project 2.0 (TLTCP 2.0) 106 Years to 115 Years. (2016). Available online at: https://1966.gov.tw/ LTC/cp-3636-38462-201.html (accessed April 20, 2018).

3. Demaerschalk BM, Hwang HM, Leung G. US cost burden of ischemic stroke: a systematic literature review. Am J Manag Care. (2010) 16:525-33.

4. Johnson BH, Bonafede MM, Watson C. Short-and longer-term healthcare resource utilization and costs associated with acute ischemic stroke. Clinicoecon Outcomes Res. (2016) 8:53. doi: 10.2147/CEOR.S95662

5. Sarker S-J, Rudd AG, Douiri A, Wolfe CD. Comparison of 2 extended activities of daily living scales with the barthel index and predictors of their outcomes: cohort study within the South London stroke register (SLSR). Stroke. (2012) 43:1362-9. doi: 10.1161/STROKEAHA.111.645234

6. Dewey HM, Thrift AG, Mihalopoulos C, Carter R, Macdonell RA, McNeil JJ, et al. Informal care for stroke survivors: results from the north east melbourne stroke incidence study (NEMESIS). Stroke. (2002) 33:102833. doi: 10.1161/01.STR.0000013067.24300.B0

7. Skolarus LE, Freedman VA, Feng C, Wing JJ, Burke JF. Care received by elderly US stroke survivors may be underestimated. Stroke. (2016) 47:20905. doi: 10.1161/STROKEAHA.116.012704 in addition to the risk factors of comorbidity and stroke severity, both severe functional impairments and cognitive impairments were found as important factors for healthcare utilizations including readmissions and re-emergency. These results regarding reserving functional abilities among stroke survivors were reckoned to be useful information for the ongoing LTC policy reform and the burden of disease for the aged society in Taiwan.

\section{DATA AVAILABILITY STATEMENT}

The raw data supporting the conclusions of this article will be made available by the authors, without undue reservation.

\section{ETHICS STATEMENT}

The studies involving human participants were reviewed and approved by Institution Review Board (No: A-ER-106-183). The patients/participants provided their written informed consent to participate in this study.

\section{AUTHOR CONTRIBUTIONS}

L-FL: study design, resource gathering, study implementation, and writing up of the manuscript. W-MW: data cleaning and statistical analyses. J-DW: consultation of the research method and resource gathering. All authors contributed to the article and approved the submitted version.

\section{FUNDING}

The study was sponsored by the Ministry of Science and Technology, Taiwan (MOST 107-2627-M-006-007 and MOST 106-2627-M-006-015).
8. Lin C-L, Hsieh S-F, Hsiao M-H, Huang JH. Predicting long-term care institution utilization among post-rehabilitation stroke patients in Taiwan: a medical centre-based study. Disabil Rehabil. (2001) 23:72230. doi: 10.1080/09638280110051376

9. Ministry of the Interior, Taiwan (MOI). Statistical Indicator: Life Expectancy. (2018). Available online at: https://goo.gl/GfAJra (accessed May 15, 2018).

10. Liu L-F, Wang W-M, Chen Y-J. The effectiveness of home services in Taiwan: a people-centered approach. Int J Environ Res Public Health. (2018) 15:2605. doi: 10.3390/ijerph15112605

11. Liu L-F, Yao H-P. Examining the need assessment process by identifying the need profiles of elderly care recipients in the ten-year long-term care project (TLTCP) of Taiwan. J Am Med Dir Assoc. (2014) 15:94654. doi: 10.1016/j.jamda.2014.07.007

12. Hwang JS, Hu TH, Lee LJH, Wang JD. Estimating lifetime medical costs from censored claims data. Health Eco. (2017) 26:e332-44. doi: 10.1002/hec.3512

13. Hwang JS, Wang JD. Monte carlo estimation of extrapolation of quality-adjusted survival for follow-up studies. Stat Med. (1999) 18:162740. doi: 10.1002/(SICI)1097-0258(19990715)18:1<1627::AID-SIM159>3.0. CO;2-D

14. Chu PC, Wang JD, Hwang JS, Chang YY. Estimation of life expectancy and the expected years of life lost in patients with major cancers: extrapolation of survival curves under high-censored rates. Value Health. (2008) 11:11029. doi: 10.1111/j.1524-4733.2008.00350.x 
15. Fang CT, Chang YY, Hsu HM, Twu SJ, Chen KT, Lin CC, et al. Life expectancy of patients with newly-diagnosed HIV infection in the era of highly active antiretroviral therapy. Qjm. (2007) 100:97-105. doi: 10.1093/qjmed/hcl141

16. Wu TY, Chung CH, Lin CN, Hwang JS, Wang JD. Lifetime risks, loss of life expectancy, and health care expenditures for 19 types of cancer in Taiwan. Clin Epidemiol. (2018) 10:581-91. doi: 10.2147/CLEP.S155601

17. Hung LC, Sung SF, Hsieh CY, Hu YH, Lin HJ, Chen YW, et al. Validation of a novel claims-based stroke severity index in patients with intracerebral hemorrhage. J Epidemiol. (2017) 27:24-9. doi: 10.1016/j.je.2016.08.003

18. Sung S-F, Hsieh C-Y, Lin H-J, Chen Y-W, Chen C-H, Yang Y-H, et al. Validity of a stroke severity index for administrative claims data research: a retrospective cohort study. BMC Health Serv Res. (2016) 16:509. doi: 10.1186/s12913-016-1769-8

19. Sung SF, Hsieh CY, Yang YH, Lin HJ, Chen CH, Chen YW, et al. Developing a stroke severity index based on administrative data was feasible using data mining techniques. J Clin Epidemiol. (2015) 68:1292300. doi: 10.1016/j.jclinepi.2015.01.009

20. Goldstein LB, Samsa GP, Matchar DB, Horner RD. Charlson Index comorbidity adjustment for ischemic stroke outcome studies. Stroke. (2004) 35:1941-5. doi: 10.1161/01.STR.0000135225.80898.1c

21. Arling G, Ofner S, Reeves MJ, Myers LJ, Williams LS, Daggy JK, et al. Care trajectories of veterans in the 12 months after hospitalization for acute ischemic stroke. Circ Cardiovasc Qual Outcomes. (2015) 8(6_suppl_3):S13140. doi: 10.1161/CIRCOUTCOMES.115.002068

22. Ku L-JE, Liu L-F, Wen M-J. Trends and determinants of informal and formal caregiving in the community for disabled elderly people in Taiwan. Arch Gerontol Geriatr. (2013) 56:370-6. doi: 10.1016/j.archger.2012.11.005

23. Tyagi S, Koh GC, Nan L, Tan KB, Hoenig H, Matchar DB, et al. Healthcare utilization and cost trajectories post-stroke: role of caregiver and stroke factors. BMC Health Serv Res. (2018) 18:881. doi: 10.1186/s12913-018-3696-3
24. Lichtman JH, Leifheit-Limson EC, Jones SB, Wang Y, Goldstein LB. Preventable readmissions within 30 days of ischemic stroke among medicare beneficiaries. Stroke. (2013) 44:342935. doi: 10.1161/STROKEAHA.113.003165

25. Greysen SR, Cenzer IS, Auerbach AD, Covinsky KE. Functional impairment and hospital readmission in medicare seniors. JAMA Int Med. (2015) 175:55965. doi: 10.1001/jamainternmed.2014.7756

26. Beard JR, Officer A, de Carvalho IA, Sadana R, Pot AM, Michel JP, et al. The world report on ageing and health: a policy framework for healthy ageing. Lancet. (2016) 387:2145-54. doi: 10.1016/S0140-6736(15)00516-4

27. World Health Organization (WHO). World Report on Ageing and Health. Geneva: World Health Organization (2015).

Conflict of Interest: The authors declare that the research was conducted in the absence of any commercial or financial relationships that could be construed as a potential conflict of interest.

Publisher's Note: All claims expressed in this article are solely those of the authors and do not necessarily represent those of their affiliated organizations, or those of the publisher, the editors and the reviewers. Any product that may be evaluated in this article, or claim that may be made by its manufacturer, is not guaranteed or endorsed by the publisher.

Copyright (c) 2021 Liu, Wang and Wang. This is an open-access article distributed under the terms of the Creative Commons Attribution License (CC BY). The use, distribution or reproduction in other forums is permitted, provided the original author(s) and the copyright owner(s) are credited and that the original publication in this journal is cited, in accordance with accepted academic practice. No use, distribution or reproduction is permitted which does not comply with these terms. 